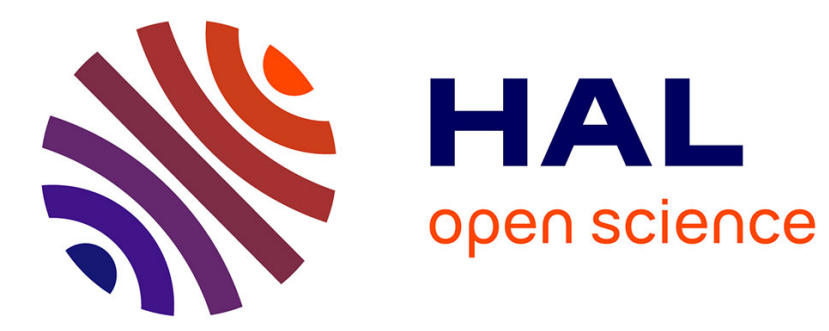

\title{
Le travail manuel masculin au cours moyen [Cent ans de recherche de cohérence]
}

Joël Lebeaume

\section{To cite this version:}

Joël Lebeaume. Le travail manuel masculin au cours moyen [Cent ans de recherche de cohérence]. Revue Française de Pédagogie, 1994, 108, pp.57 - 71. 10.3406/rfp.1994.1258 . hal-03558848

\section{HAL Id: hal-03558848 \\ https: / hal-univ-paris.archives-ouvertes.fr/hal-03558848}

Submitted on 5 Feb 2022

HAL is a multi-disciplinary open access archive for the deposit and dissemination of scientific research documents, whether they are published or not. The documents may come from teaching and research institutions in France or abroad, or from public or private research centers.
L'archive ouverte pluridisciplinaire HAL, est destinée au dépôt et à la diffusion de documents scientifiques de niveau recherche, publiés ou non, émanant des établissements d'enseignement et de recherche français ou étrangers, des laboratoires publics ou privés. 


\section{Le travail manuel masculin au cours moyen}

Cent ans de recherche de cohérence

M. Joël Lebeaume

\section{Citer ce document / Cite this document :}

Lebeaume Joël. Le travail manuel masculin au cours moyen. In: Revue française de pédagogie, volume 108, 1994. pp. 57-71; doi : https://doi.org/10.3406/rfp.1994.1258

https://www.persee.fr/doc/rfp_0556-7807_1994_num_108_1_1258

Fichier pdf généré le 04/02/2020 


\begin{abstract}
In this survey, conditions of availability of handicrafts for boys in the last two years of primary school are analysed. In this perspective, the emergence, development, transformation and then disappearance of this subject matter between 1882 and 1985, is reported. During this period, the way of naming this subject changed several times, in its struggle for recognition. The survey also deals with an analysis of prescribed contents and in particular shows the difficulty of elaboration of a consistent and specific scheme fo 10-12 years ola boys, supposed to be too big for hand exercices and too small for sheer technical education, though in 1985 technology was introduced. The variations of content reveals the conflict of references implied by didactic integration of social pratice.
\end{abstract}

\title{
Résumé
}

Cette étude vise à analyser l'existence du travail manuel dans l'organisation pédagogique de l'école primaire, plus particulièrement dans les cours moyens des écoles de garçons. Pour ce faire, elle s'applique d'abord à décrire la genèse, les évolutions, les transformations puis la disparition de cette discipline née en 1882 et disparue en 1985. Pendant cette période, les appellations génériques de travail manuel, travaux manuels, travaux manuels éducatifs, éducation manuelle et technique ou activités techniques... témoignent des différentes tentatives de légitimation de cet enseignement. L'étude porte parallèlement sur une analyse des contenus prescrits et montre notamment la difficulté de construction d'un ensemble cohérent, spécifique aux garçons de 10-12 ans jugés longtemps, trop grands pour les exercices de la main et trop petits pour une première éducation technique désintéressée, ce que tente d'assurer la technologie introduite en 1985. Mais, sans doute plus que toute autre discipline, les oscillations de son contenu font apparaître le conflit des références qu'implique l'intégration didactique des pratiques sociales. 


\title{
Le travail manuel masculin au cours moyen
}

\section{Cent ans de recherche de cohérence}

\author{
Joël Lebeaume
}

Cette étude vise à analyser l'existence du travail manuel dans l'organisation pédagogique de l'école primaire, plus particulièrement dans les cours moyens des écoles de garçons. Pour ce faire, elle s'applique d'abord à décrire la genèse, les évolutions, les transformations puis la disparition de cette discipline née en 1882 et disparue en 1985. Pendant cette période, les appellations génériques de travail manuel, travaux manuels, travaux manuels éducatifs, éducation manuelle et technique ou activités techniques... témoignent des différentes tentatives de légitimation de cet enseignement. L'étude porte parallèlement sur une analyse des contenus prescrits et montre notamment la difficulté de construction d'un ensemble cohérent, spécifique aux garçons de 10-12 ans jugés longtemps, trop grands pour les exercices de la main et trop petits pour une première éducation technique désintéressée, ce que tente d'assurer la technologie introduite en 1985. Mais, sans doute plus que toute autre discipline, les oscillations de son contenu font apparaître le conflit des références qu'implique l'intégration didactique des pratiques sociales.

\section{INTRODUCTION}

Cette étude (1) est une contribution à l'histoire des disciplines qui permet aujourd'hui de mieux identifier la spécificité des pratiques scolaires et met en évidence le processus complexe de scolarisation des savoirs comme le définissent André Chervel et Jean Hébrard (2). Remarquons que nous qualifions le travail manuel de discipline dans son acception essentiellement administrative et institutionnelle apparente dans la qualification des enseignants et leur formation spécifique (3). Ainsi, il conviendrait sans doute, de privilégier le concept proposé par Jean Hébrard, d'ensemble de pratiques cohérentes (4), que recouvre l'intitulé générique de travail manuel.
Commencer l'examen du travail manuel à partir de 1882 occulte en partie les étapes de la scolarisation des pratiques professionnelles dont nous supposons qu'il émane. Ce processus de déprofessionnalisation est associé à la nécessité de communication qu'engendre, dès le XVIII', la formation des artisans et qui, par souci de généralisation deviendra peu à peu un savoir sur un savoir faire plutôt qu'un savoir faire.

Néanmoins l'école définie par les lois laiqques poursuit ce traitement scolaire sous le poids, selon l'expression de Pierre Arnaud (5), de * l'orthodoxie scolaire » conférant à la discipline ses attributs scolaires, ses rites initiatiques, ses signes distinctifs. Pour ce qui concerne le travail manuel nous ne serons pas surpris d'observer ces 
opérations de mise en conformité des activités proposées aux élèves avec la norme implicite des activités scolaires, éducatives ou formatrices. Pour Pascale Rougier-Pintiaux (6), originellement, l'école primaire n'a pu concevoir cet enseignement qu'en le transformant en travail manuel éducatif, distinct d'un enseignement professionnel à l'intention des classes populaires. Faut-il alors supposer que toute cohérence des contenus repose fondamentalement sur leur fonction éducative ? Ou bien convient-il d'expliciter ce mode d'existence afin d'éclairer toute tentative d'introduction et d'insertion de pratiques nouvelles dans l'école?

Afin d'éviter une dispersion, notre attention s'est plus particulièrement portée sur le cours moyen en raison de la charnière qu'il représente entre l'école primaire et le cours supérieur ou le collège et car ce niveau constitue le lieu de développement d'une discipline à l'école primaire. De plus nous avons observé plus attentivement le travail manuel masculin dont les prescriptions, avant la généralisation de la mixité, sont citées dans les textes destinés aux écoles de filles.

Le travail de reconstitution a été effectué à partir de l'analyse des prescriptions sous leur forme institutionnelle que sont les instructions officielles ou sous leur forme commerciale que sont les manuels. Nous avons été conduit à examiner par ailleurs les articles parus dans les revues pédagogiques au cours de cette période. II s'agit donc plus particulièrement de l'étude du discours sur le travail manuel, distant du travail manuel effectivement enseigné. L'évolution de ce discours permet de distinguer les différentes étapes de la vie de cette activité scolaire et les questions qu'elle suscite. Nous suivrons ainsi cette vie du travail manuel, le parcours séculaire de ses formes scolaires puis présenterons quelques propositions interprétatives de cette évolution.

\section{CONCEPTION ET NASSANCE}

Les lois scolaires n'ont pas imposé ex nihilo le travail manuel à l'école. Elles ont assuré une généralisation de tentatives, menées notamment à Paris et à l'étranger, en particulier en Suede et aux États-Unis. En France, cette phase d'élaboration est marquée par la ténacité de l'inspecteur général G. Salicis dans une période où s'établit peu à peu un consensus à propos de la pratique du travail manuel à l'école. Á cet égard, trois courants de pensée complémentaires sont identifiables, celui de l'idéal d'une culture " harmonique (7) associant éducation physique et éducation intellectuelle, celui du développement de l'enfant qui se concrétise par les expériences des jardins d'enfants et des salles d'asile que prônent Pestalozzi, Fröbel, Pauline Kergomard ou Maria Montessori, celui enfin de la formation professionnelle et de l'apprentissage qui tente de retarder l'échéance sociale de la scolarisation tout en désignant des écoles spécialisées. Les écoles d'apprentis de Gréard proviennent en effet de la double préoccupation d'une part, d'assurer une véritable formation professionnelle et d'autre part, de protéger les enfants pour lesquels l'apprentissage se confond alors, trop souvent, avec initiation alcoolique et délinquance (8). Ainsi, dans les années 1880 se réalise le vœu révolutionnaire de prise en charge sociale des enfants. Étre social singulier, l'enfant existe aussi en tant qu'être biologique et psychologique distinct de la simple réduction géométrique de l'adulte, idée que renforceront les études de Binet à la fin du siècle.

Au plan des structures, l'institutionnalisation de l'apprentissage assure en fait le découpage de l'éducation en trois niveaux spécialisés et hiérarchisés que sont l'école maternelle, l'école primaire et, au delà du cours moyen, le cycle terminal de préparation à la vie. Or cette spécialisation des étapes et des apprentissages qui leur sont dévolus risque de mettre en péril le redressement économique souhaité au lendemain de la défaite militaire. G. Salicis craint en effet que l'école moyenne contrarie le déterminisme naturel en donnant aux enfants des envies, des pratiques, trop distantes des rôles pour lesquels ils sont destinés. Aussi, l'argument essentiel qu'il brandit constamment est celui du développement du goût pour le travail manuel afin de réaliser "l'armée industrielle $*(9)$ aux $*$ bras intelligents $\nsim$ dont la France a besoin. G. Salicis revendique ainsi l'introduction précoce du travail manuel afin de maîtriser la transformation des instincts de jeu en instincts de travail. Cette proposition se heurte à Gréard qui, par souci d'une scolarisation massive, est hostile à toute dispersion et veut répondre à la demande sociale dont les aspirations lui semblent opposées à toute approche professionnelle, fusset-elle lointaine. Cependant, il tolère le travail manuel après l'Ecole, le jeudi et le soir lorsque les jeunes garçons sont oisifs. 
Parallèlement, avec le soutien et la conviction de Corbon et Tolain, G. Salicis (10), met en œuvre en 1872 une première forme de travail manuel dans l'école dont l'exemple de la rue Tournefort reste un modèle. L'expérience étendue dans dix sept écoles parisiennes prouve l'intérêt d'une telle pratique scolaire et permet d'argumenter en faveur de cette généralisation car " il serait impossible de lui trouver un inconvénient, et qu'il n'a, au contraire, que des avantages * (11).

\section{LANCEMENT}

La loi organique de 1882 propose alors cette généralisation au pays et le travail manuel devient enseignement obligatoire étroitement solidaire de l'éducation physique conçue comme une préparation militaire. Fondé sur un finalisme sexuel incontesté, évident, ce travail manuel est distinct pour les fillettes et les garçons. Pour les filles, il s'agit avant tout de contribuer à la formation de la mère et de l'épouse garante de l'élément de la nation que constitue la famille. *Préférer une pièce mal mise plutôt qu'un trou bien fait $n$, « un intérieur plutôt qu'un gîte " (12) guide les travaux d'aiguilles prescrits. Pour les garçons, développer l'habileté manuelle et le goût pour le travail manuel sont les impératifs essentiels de la recherche de contenus ou d'exercices spécifiques à ce segment de l'École. Imposer une " première orthographe de la main ", parvenir "à mettre le compas dans l'œil " fixent les progressions des travaux de bois et de métal, des exercices de modelage, de la section enfantine au cours supérieur en vue du développement des compétences communes à tous les métiers.

Cette compétence, que l'on qualifierait aujourd'hui de transversale, trouve sa cohérence plus particulièrement dans l'explicitation de la géométrie des objets fabriqués. Celle-ci se réfère, d'une part, à leur représentation qu'est le dessin industriel et d'autre part, à leur réalisation qui provient inévitablement de la composition de plans et de volumes. Mais ce point de vue privilégié s'articule également avec les exercices proposés dans les Kindergärten de Fröbel (13). C'est là le point de cohérence interne des contenus proposés qui peuvent s'établir selon une progression à l'intérieur de l'école, dans la perspective concentrique de l'organisation pédagogique. Cette progression peut s'observer selon trois axes : a) l'axe géométrique poursuivant les points et les lignes de l'école maternelle, à savoir le plan dans la section enfantine, le volume ou le solide simple au cours élémentaire et le volume complexe ou le solide composé au cours moyen.

b) l'axe des matériaux, d'abord les fils souples en section enfantine et les feuilles de papier au cours élémentaire puis, l'usage de matériaux plus résistants tels les tiges de bois et le fil de fer au cours moyen enfin, le travail en masse du métal et du bois au cours supérieur.

c) l'axe de la dextérité fondée à la fois sur le jeu des coordinations et la force des enfants ce qui se traduit par une individualisation des segments de la main, puis du contrôle œil main et enfin du contrôle tonique.

II s'agit d'un ensemble d'exercices gradués, allant du simple au composé, adaptés à l'école primaire car en prolongement des activités des tout petits et préparatoires à celles des cours supérieurs. Cette organisation est une traduction de la vision associationniste de la psychologie, apparente aussi dans les exercices systématiques de l'apprentissage du toucher de Maria Montessori (14) et de l'ABC technique recherché dans l'éducation de Gertrude par Pestalozzi (15).

La méthode d'enseignement se caractérise par la "méthode des objets utiles" définie par Schmitt (16) qui s'oppose à a la méthode des éléments techniques $\%$. Le souci majeur est la confrontation des exercices scolaires avec les objets de l'environnement. Ainsi, les travaux d'assemblage de tiges de bois sont associés à la construction des échelles, les travaux de treillage de fil de fer au garnissage de paniers à salade ou de cages. Elle s'oppose à la méthode des éléments techniques propre à la formation préprofessionnelle se focalisant davantage sur la rigueur des opérations de corroyage ou de limage dans la réalisation systématique de modèles partiels.

Cette phase de lancement se caractérise par la résolution du dilemme école-atelier qui distancie le travail manuel à l'école de toute prétention professionnelle proposant des activités adaptées aux enfants (en particulier à leur force car le travail manuel demeure avant tout une épreuve physique) et en préconisant une méthode, celle des objets utiles, assurant le lien avec des pratiques sociales de référence (17), ... mais décadentes dans cette fin de siècle alors que l'industrialisation et la mécanisation sont en plein essor. 


\section{DÉCLN ET NOUVEAU TRAVAll MANUEL}

Ces prescriptions sont répétées, reformulées, présentées à maintes reprises par G. Salicis qui regrette que l'Etat n'accompagne pas ses intentions des équipements souhaités. Le plan de formation, par ailleurs, ne permet d'assurer que la formation des maîtres des cours complémentaires. La mort de Salicis en 1889 laisse orphelin ce travail manuel qui trouve un tuteur en R. Leblanc, inspecteur général, fidèle assesseur de Salicis depuis 1885. Leblanc, dont l'ambition première est de vulgariser les sciences pour que chaque citoyen soit à même de lire et d'interpréter les revues scientifiques de l'agriculture, métamorphose le travail manuel. Cette transformation s'articule aussi avec l'évolution interne des programmes qui, d'après P. Ognier (18), tient compte des critiques envers la formation militaire et des soucis de limitation du surmenage scolaire. La gymnastique privilégie alors les exercices physiques et les jeux scolaires. Ainsi, dès 1891, R. Leblanc expérimente le travail manuel inscrit au programme officiel de la ville de Paris. Présent, cette fois, au chapitre de l'éducation intellectuelle, il devient par conséquent éducatif et se conforme aux règles du fonctionnement scolaire.

Le caractère géométrique est exclusivement développé et ce travail manuel est un instrument pédagogique. Pour Leblanc \& les travaux manuels de l'école élémentaire sont à l'enseignement des notions de sciences mathématiques ce que sont les expériences à l'enseignement des sciences physiques et naturelles (...) $\approx(19)$. Un exercice manuel d'école « doit exercer l'œil et la main, en même temps que son attention, son intelligence, son goût et son adresse ; il faut, en outre, qu'il soit peu coûteux, en rapport avec les forces physiques de l'élève (...). Mais cela ne suffit pas (...) il faut qu'il prête son concours à l'éducation intellectuelle en apportant à la partie scientifique (dessins, formes géométriques, calcul) le concret qui lui fait si souvent défaut dans l'enseignement ordinaire ». Arguant qu' ' un travail bien tracé est un travail à moitié fait ", R. Leblanc gomme la dimension technique du travail manuel.

Dans cette perspective, le travail manuel scolarisé s'écrit sur un cahier particulier dans lequel l'élève copie l'exercice, trace la forme aux instruments, la réalise en papier ou carton, la fixe sur la page puis note les observations, calculs, définitions... tout en apportant le plus grand soin à la réalisation progressive de cet album. Le modelage se transforme également en une forme d'écriture à l'ébauchoir décomposant méthodiquement les modèles à reproduire. Peu à peu, le modelage disparaît des programmes au profit du coloriage permettant de rendre aux solides représentés leur volume.

Cet apprentissage systématique de la raison géométrique couronné lors de l'exposition universelle de 1900 n'est qu'une illustration savante des formes, des figures, des règles de la géométrie et de la stéréotomie. Dans cette nouvelle version baptisée "le travail manuel sans atelier ", la méthode des objets utiles est remplacée, non pas par la méthode des éléments techniques, mais par celle des éléments géométriques.

Ainsi naît le paradoxe du travail manuel qui ne prend plus référence ni sur le travail ni sur le geste manuel et dont la fonction essentielle est de développer la justesse de l'œil. En s'adressant d'une façon privilégiée à l'œil, il se rapproche de l'esprit et légitime l'épithète éducatif qui l'accompagne. Cette opération de distanciation, à la fois de la relation physique avec le matériau et du geste productif de l'action technique, est la caractéristique apparente de ce traitement scolaire.

Auxiliaire de la science, notamment de la géométrie, il participe à $\propto$ l'enseignement manuel et expérimental ^ du nom du périodique (20) dirigé par R. Leblanc. Certains, par exemple Girauld, inspecteur d'académie de l'Aude souhaiteraient son extension à toutes les sciences. Mais peu à peu le travail manuel est oublié avec le décès de Leblanc en 1909 et la grande guerre. Les instructions de 1923 officialiseront cet oubli.

\section{L'OUBLI DU TRAVAIL MANUEL}

Les instructions et le nouveau plan d'études de 1923 ne sont, comme les historiens contemporains (21) l'observent, que des instructions de recentrage qui * restent fidèles aux principes des fondateurs $n$. II s'agit comme l'annoncent les nouvelles prescriptions de " simplifier et de graduer les programmes, de vivifier les méthodes, de coordonner les disciplines: préciser, simplifier, vivifier et ordonner, tel a été notre dessein » peuton lire dans leur présentation. C'est aussi une remise en cause profonde de la méthode d'instruction. La mémorisation excessive est 
dénoncée car elle "entraîne des souvenirs mutilés et confus " et la "leçon de choses ", en classe et en promenade, est mise en exergue privilégiant l'activité non livresque d'observation et d'expérimentation.

Les instructions de sciences physiques et naturelles tentent de promouvoir un enseignement "simple, concret, expérimental, pratique". Celles spécifiques à la science mathématique prônent également le concret, l'action de l'élève, la mise en œuvre de la méthode inductive et intuitive. En dessin, les tendances de 1909 sont réaffirmées privilégiant l'observation, l'exécution en perspective car la représentation d'objets éduque l'observation vigilante, force la mémoire visuelle qui prépare la réalisation de croquis. Le dessin géométrique, * auxiliaire précieux de l'enseignement scientifique et instrument indispensable à toute profession n est plus spécialement destiné aux écoles de garçons (CM). II permet de faire comprendre l'usage des instruments géométriques et les éléments du dessin géométral (projection et cotation).

Ces descriptions disciplinaires recouvrent en grande partie les grandes thèmes du travail manuel éducatif de Leblanc et ses finalités morales. Le programme scientifique, en effet a " pour objet de fournir à l'enfant les notions indispensables dans la plupart des professions ». II doit * armer les travailleurs, augmenter le rendement de leur activité productrice $\%$, en définitive former, enfin, cette armée de travailleurs aux bras intelligents. Mais cette préoccupation professionnelle, ne se centrant que sur la géométrie des objets, n'est que très partielle.

Le travail manuel est ainsi étroitement associé, intégré, aux disciplines scientifiques et perd son originalité. * II sera d'autant plus en honneur qu'il portera moins ombrage aux autres disciplines " supposent les instructions. Passé dans l'ombre, en filigrane, il n'est plus en amont de la connaissance des figures géométriques mais c'est un " secours "; de même il ne matérialise plus les phénomènes des sciences, il valorise les expériences scientifiques.

Toutefois, ces instructions de 1923 laissent présent un aspect pratique, celui de la préparation aux petits travaux domestiques quotidiens a pour se tirer d'embarras ". Mais quelle est la valeur scolaire de ce savoir lorsque le travail manuel fait partie de l'éducation intellectuelle ? Ainsi, en devenant surtout l'accessoire des disciplines il devient accessoire.

\section{LA NAISSANCE DES ACTMITES MANUELLES}

Dans les années 30 , hormis la dimension domestique dominante dans les activités de préparation à la vie, la référence exclusive des contenus est celle des autres disciplines scolaires au statut majeur. Le travail manuel est donc un moyen pédagogique facilitant l'observation, la mise en évidence des phénomènes, leur généralisation à partir des supports concrets qu'il impose. Cette pédagogie par le concret est notamment promue par la revue * le travail manuel, les sciences expérimentales et le cinéma à l'École " (22) qui apparaît la seule publication nouvelle de cette période. L'équipe de rédaction (23) se définit dans la lignée des propositions de $\mathbf{R}$. Leblanc et propose chaque mois des exercices de géométrie, de découpage de bois, de croquis coté, de travail du fil de fer. Les enfants sont ainsi conduits, par exemple pour circonscrire le carré, à l'appréhender par des pliages, par sa construction aux instruments, par la réalisation de son * squelette * en fil de fer, par sciage de contreplaqué... Ce nouveau travail manuel qualifié d'« attrayant » par Plicque, pour $\alpha$ instruire en intéressant et en amusant les enfants tout en s'adressant à leurs facultés créatrices (24), se caractérise par la confection d'objets simples et utilisables. Ce sont majoritairement des jouets tels que chariots ou animaux... en bois et des objets tels que coquetier, abat-jour... en fil de fer.

La formation suggérée se résume ainsi à la préparation aux apprentissages scolaires et, d'une façon moindre, à la préparation aux tâches quotidiennes de la vie privée. Tout en louant le travail, cette éducation occulte totalement la préparation à la vie professionnelle, au métier, facette qui conserve toutefois ses partisans. Notamment, Kula et Bocquillon (25) proposent de relier les deux facettes divergentes du travail manuel, d'associer les travaux de papier plié à la réalisation d'objets vrais, d'assurer une cohérence entre contenus scolaires et références sociales. Soucieux d'éviter que * la plume ne chasse l'outil *, que l'éducation intellectuelle ne fasse disparaitre la devise de jadis * vive labeur * (26), dans un espoir d'une France rurale non soumise * au guet-apens de la 
grande industrie $\infty$, cet industriel et ce directeur d'école proposent d'avoir un métier que l'on aime, condition essentielle du bonheur. Assurés de la valeur éducative des travaux menés à la main, ils proposent une adaptation des contenus géométriques à un acheminement et une préparation à la production. Du cours préparatoire au cours moyen, les contenus sont organisés autour de la ferblanterie qui, selon les auteurs, "doit être considérée, non comme une préparation au métier de ferblantier, en partie disparu, mais comme une méthode incomparable de culture manuelle générale, préparatoire à tous les métiers manuels ». Cette formule nouvelle est, en réalité, une variation des exercices de géométrie qui prennent leur sens dans la réalisation terminale de gobelets, entonnoirs, boîtes à lait, filtres à café, burettes à huile, brocs, seaux...

Cette proposition qui ne parvient pas à convaincre les universitaires, au désespoir de leurs auteurs, reste une tentative de conciliation entre les deux versants du travail manuel, formation intellectuelle et formation professionnelle. Elle montre surtout les limites que l'école impose aux pratiques de référence. Pour Kula et Bocquillon, l'objet réalisé, la démarche d'enseignement se définissent par rapport à l'apprentissage professionnel. Kula a en effet fondé le premier « AtelierÉcole de Préapprentissage * (27), " intermédiaire entre l'École et l'Atelier, sorte de plan incliné, ou de pont entre l'une et l'autre (28). Dans cette perspective, le seau en fer blanc s'oppose radicalement à l'éléphant en bois de Plicque car ces deux objets scolaires n'ont pas la même signification. Bien que ces deux objets soient des traductions pour les élèves du savoir à transmettre, l'un se veut authentique de la réalité professionnelle alors que l'autre éloigne la frontière de la réalité sociale en épurant l'objet de son contexte de réalisation pour ne montrer que son essence géométrique.

Après les modifications du système éducatif consécutives à la crise économique et au Front Populaire, l'accent est porté sur la liberté de l'enfant, sur son activité spontanée, légalisant ainsi la pénétration des méthodes d'éducation nouvelle en particulier dans les activités dirigées. La contraction activités manuelles (activités dirigées et travail - manuel), qui se généralise dès cette date, privilégie le point de vue de l'enfant * artisan de sa propre éducation en méme temps que son sens social se développe $\nsim(29)$.
Durant les trois heures hebdomadaires ainsi réservées à " ces modes d'éducation plus libre, moins asservies aux méthodes qui s'imposent à l'intérieur de la classe " (30), les classes promenades, la constitution de collections, les jeux... consacrent une place importante à l'activité manuelle. Par ailleurs, l'encouragement aux fêtes scolaires et le souhait de a faire réaliser des travaux utiles, faire confectionner des objets utilisables * permettent de s'orienter vers la réalisation d'objets " offrables " et " vendables ". Cette orientation ouvre la voie de ces multiples fabrications qui orneront les tables d'exposition des coopératives scolaires.

Les activités manuelles sont, à cette époque, classées exhaustivement selon les matériaux et illustrées par d'abondantes collections (31) de livrets. C'est en fait du travail manuel au pluriel, des travaux manuels. Mais dans ces activités dirigées présentées par ces catalogues d'objets scolaires tels que fleurs en papier, bracelets de perles, colliers de papier, maquettes... il n'y a plus explicitement de progression, seulement une juxtaposition d'activités variées.

Les textes de la période d'occupation, quant à eux, renforcent les activités de préparation à la vie courante à l'intérieur et en plein air. Détachage, réparation d'un livre..., remise en état des terrains de jeux et de sports ... et des travaux de jardinage se juxtaposent aux réalisations de sousverre, de mastiquage de fenêtre, de bouchage et débouchage de bouteille (!), de pose d'un verrou, de confection de nœuds ou d'aiguisage de couteaux. Aux côtés de ces travaux d'entretien matériel de l'école sont toujours présents les petits travaux décoratifs, ces transformations de matériau tantôt à dominante géométrique, tantôt à dominante décorative. À la fin de la guerre, ces préoccupations liées à la préparation à vivre en autarcie dans les villes et les campagnes que l'occupation peut expliquer, sont minorées. La nouvelle organisation scolaire attribue dorénavant une nouvelle fonction au cours moyen, préparatoire à la classe de $6^{\circ}$ mais dont l'examen d'accès, ou la certification de sortie, ne valide pas le travail manuel.

Ainsi, au lendemain de la seconde guerre (32), le travail manuel est toujours présent avec une nouvelle cohérence. Si la méthode d'enseignement qu'il portait en son sein s'avère plus ou moins intégrée dans les activités géométriques et les activités scientifiques, la partie pratique renaît en 
se précisant activités manuelles. Variété des matériaux, variété des objets témoignent d'un élan de liberté, des élèves et des maîtres mais présentent aussi le risque d'exclusion scolaire reposant sur le paradoxe même de la préparation à la vie courante qui peut se confondre avec une activité péri-scolaire car post-scolaire et, peut-être a-scolaire.

Le travail manuel met ainsi entre parenthèses le versant professionnel de la préparation à la vie en reconnaissant la rupture entre la vie professionnelle et la vie privée. Cette orientation se focalise alors sur l'activité de l'élève en raison du souci d'engagement de l'enfant dans sa réalisation afin de développer ses capacités. Ce modèle d'éducation nouvelle caractérisé par la motivation, la création autonome, la mise en cuvre collective sera officialisé par le tiers temps pédagogique des textes de 1969.

\section{MATURITÉ ET DÉCLIN DES TRAVAUX MANUELS}

La période 1950-1970 est marquée par ce que l'on pourrait appeler la * vie active * du nom de l'association pour le développement du travail manuel dans l'éducation fondée par Albert Bcokholt (1946). R. Cousinet décrit cette activité : * l'enfant, honnête ouvrier, construit l'œuvre désirée avec les éléments qu'il a à sa disposition et dont il connaît et apprend à connaître les possibilités, il résoud le problème qu'il s'est posé avec les données qui rendent le problème possible $*(33)$.

Cependant, les revues pédagogiques ne suggèrent que rarement cette démarche. Seuls, parfois, sont exposés les savoir-faire de techniques variées ou des descriptions de réalisation sans commentaire pédagogique comme si, par définition, la réalisation de ces modéles suffisait à apporter l'aspect éducatif accordé aux travaux manuels. Coupe à fruits en vannerie, sandales de raphia, sacs, corbeilles de toilette, cadres de cartes postales ... sont ainsi suggérés aux maîtres. En revanche, ces revues continuent à développer, dans la perspective du dessin de mémoire et du dessin géométral; les objets à caractère décoratif par collage de formes géométriques sur des silhouettes ou les objets à caractère géométrique telles que construction d'une maquette d'usine ou construction de chapeau conique qui ne sont, en somme, que des variations sur les volumes.

Ainsi dans la littérature autorisée, les travaux manuels se confondent toujours avec le dessin géométral ou les activités géométriques tandis que se développent, parallèlement, des activités de fabrication promues par la commercialisation des produits scolaires, carreaux ou récipients en céramique, sujets, vases ou médaillons en plâtre, coffrets, coquetiers ou pieds de lampe en bois ... que les enfants terminent (34). C'est l'époque de l'enthousiasme de la main créatrice, correspondant à la phase esthétique qu'identifie Eric Plaisance (35) pour l'École Maternelle et qui transparaît également dans les textes relatifs aux travaux manuels éducatifs du second degré (36).

Les années 60 correspondent à la mise en œuvre de la grande réforme du système éducatif qui prolonge la scolarité obligatoire jusqu'à 16 ans. L'école primaire devient un maillon intermédiaire, sans validation pratique et sociale à son issue. Après 1968, l'école peut prendre son temps et ajuster ses exigences aux capacités psychologiques des élèves, aux besoins des enfants. L'éveil, nouveauté de l'organisation hebdomadaire, inclut le travail manuel dont les instructions demeurent, néanmoins, toujours celles de 1923. Bien qu'a priori, tout soit matière à éveil de la pensée, la distinction des disciplines d'éveil permet aux plus fragiles, dont le travail manuel, de revendiquer leur spécificité. La dimension fabricatoire apparait alors la caractéristique fondamentale de ce travail manuel qui, depuis 1923, était assimilé à l'instrument pédagogique des disciplines où l'observation active s'opposait à l'écoute docile d'une leçon. Présent en tant que discipline d'éveil, il trouve alors son originalité dans la fabrication d'objets décoratifs ou utilitaires. Construire un cerf volant, réaliser un petit train en bois, tresser du papier, modeler du papier mâché, tendre des fils, transformer de la corde armée, tisser des perles... sont des activités caractéristiques qui ne sont pas étrangères aux préoccupations de la société des loisirs naissante.

Cet engouement fabricatoire, quelque peu hétéroclite, suscite des critiques sous l'expression péjorative de bricolage. En effet les quelques descriptions montrent qu'il n'y a ni observation, ni activité, ni créativité de l'élève car, très souvent, le maître montre et l'élève exécute. Ce qui est en cause c'est le caractère éducatif de ces activités 
qui apparaissent proches d'un « dressage artisanal s (37) ou d'une simple occupation, un exercice manuel opposé à une activité manuelle.

\section{NAISSANCE ET MATURITÉ DES ACTMITES MANUELLES ÉDUCATIVES}

Cette critique conduit à une réflexion, en particulier dans les écoles normales (38), qui souhaite structurer les apprentissages et définir le qualificatif " éducatives " adjoint aux activités manuelles. En réaction, elles se fondent sur l'étroite relation entre éveil de la pensée et activité manuelle (39). Les activités manuelles se veulent " imbriquées dans les autres activités de l'école élémentaire » afin "d'inciter les élèves à la réflexion sur les processus logiques qui accompagnent, déterminent l'activité manuelle ou en sont les prémisses ou les conséquences * (40). Les propositions d'activités définissent une méthode nouvelle, que l'on peut qualifier « des éléments logiques ", appliquée dans deux directions.

La première porte plus particulièrement sur la représentation des actions bimanuelles coordonnées (41), considérées comme des opérations concrètes. II s'agit de faire, dire, puis représenter une tâche, une action, de décrire un processus en définissant le programme d'actions, l'algorithme (42). Ainsi l'analyse des diverses actions décompose ces actes en règles; coder une tresse à trois brins permet d'en décrire, d'abord la règle d'entrelacement puis de la réinvestir et de la généraliser à des tresses à $n$ brins; plier du papier devient un support de repérage de la localisation des plis et de la chronologie des opérations. Mais le travail manuel ne se limite pas à cette dimension mathématique qui est abondamment étudiée dans les IREM et dont l'ouvrage " mathématiques pour la tête et les mains " donne une illustration (43).

En effet, la seconde direction de cette méthode des éléments logiques considère la finalité de l'action et la logique de l'organisation de l'objet et des conditions de réalisation (44). Ainsi, des activités permettent d'envisager, autour d'une activité thématique d'éveil, l'activité de réalisation d'objets. Celle-ci prend alors le sens que lui donne l'interdisciplinarité. L'usage d'outils, le choix d'organisation et de méthode remplacent l'apprentissage gratuit et systématique de techni- ques disparates. Activité spontanée, analyse, investissement sont proposées comme les étapes essentielles de l'intériorisation des opérations concrètes, de la construction des structures progressives de la pensée et du développement et de la manifestation de la créativité.

Les décompositions algorithmiques des techniques d'assemblage et la justification des choix et des étapes associés à un objet fabriqué légitiment le caractère éducatif de ces travaux dans le système scolaire en lui conférant un statut pédagogique (45) car cette intelligence de l'action se veut l'articulation indispensable avec les mathématiques. Psychologisation (46) et mathématisation des activités manuelles leur confèrent l'attribut éducatif, condition d'homologation dans le système scolaire.

Toutefois, la pratique abondante, voire désordonnée, de techniques demeure dans les classes sous forme d'ateliers décloisonnés qui prennent comme support de motivation des enfants ou de répartition des élèves les activités manuelles. Moyen de décloisonnement, les activités manuelles pratiquées en ateliers ne sont plus activifés d'éveil mais réalisations libres ou commandées. Ce divertissement qui n'est pas une véritable distraction, cette récréation qui ne suggère pas une re-création témoignent d'un intérêt prioritaire accordé aux initiations aux techniques manuelles, encouragées par la campagne ministérielle de revalorisation du travail manuel menée de 1973 à 1981.

À partir des années 1980, la dimension strictement manuelle s'estompe progressivement au profit de la référence technique. Si, au Collège, l'éducation manuelle et technique se structure autour de la fabrication d'objets techniques dans laquelle la vocation expressive n'est plus majeure, l'école primaire maintient sa centration sur l'enfant et propose une formation méthodologique et, pour la première fois, une formation technique. Celle-ci, formulée dans le style qu'impose l'engouement pour la pédagogie par objectifs, consiste à rendre l'élève capable de a mener à bien des travaux diversifiés * (47) c'est-à-dire savoir choisir et utiliser des matériaux et des outils, ce qui requiert une connaissance de ces instruments et de ces matériaux. La formation méthodologique correspond aux étapes précédant la mise en forme des matériaux c'est-à-dire développer les capacités de conception, d'organisation, de recherche, de représentation, de critique, d'investissement. 
Comment faire et faire sont ainsi associés dans une méthode qui se définit, celle du " projet technique $»$.

Les illustrations de ces prescriptions (48) montrent les relations interdisciplinaires dans la pédagogie de l'éveil en présentant des activités manuelles à dominante : dominante géométrique de pavages de plan obtenus par déplacement de pochoirs, par tapisserie, par broderie, par nouage, par broderie ou par collage de formes en papier ; dominante créative avec la recherche systématique de modifications des matériaux tels le papier ou l'aluminium à repousser ou variations sur le point devant; dominante physico-chimique de découverte des conditions d'obtention des confitures, du fromage, d'utilisation des propriétés de l'amidon à la fois dans les préparations culinaires et les transformations des caractéristiques des étoffes ; dominante technique centrée sur un procédé comme le moulage. La dominante majeure est physico-technologique se centrant sur la structure du matériau comme le carton ou bien sur le principe de fonctionnement d'objets par la réalisation de maquettes de jouets animés, 200trope, appareil photographique, moulin, péniche, machines à tisser.

Cette dominante assure, au cycle moyen, le glissement de l'activité manuelle-éveil de la pensée vers un éveil technique qui a pour but de « permettre à l'enfant de comprendre et de fabriquer les objets matériels de son environnement " (49). Le plaidoyer pour la culture technique permet de proposer à l'enfant "l'accès à une pensée technique * qui, selon les promoteurs implique d'avoir d'une part \& un authentique vécu de fabrication * et d'autre part une possibilité d'intervention sur des * objets ouverts ». II s'agit de permettre à l'enfant de fabriquer, d'agir, d'inventer, de tâtonner à propos d'objets ludiques ou utilitaires. Ces vrais objets réalisés dans les conditions matérielles des classes non dotées d'équipement portent la trace des maladresses de leurs auteurs. Bien que les opérations soient effectuées principalement avec un outillage manuel, les procédés envisagés transposent les principes de production de série. Débiter quatre cubes identiques pour réaliser un calendrier perpétuel, réaliser l'impression multiple de visages de poupées en utilisant des butées, des pochoirs, des organisations, permettent une première familiarisation avec les procédés artisanaux ou industriels. Cet éveil technique propose un contenu spécifique qui ne se référe plus aux métiers, à l'action mais à la transversalité des principes de production, notamment les moyens de mise en position des éléments et les modèles de machines qu'ils supposent.

Cette innovation de contenu qui prend référence sur un savoir réservé aux élèves des sections spécialisées de l'enseignement technologique peut apparaître en rupture avec les savoirs conformes de l'école de l'excellence qui s'est distinguée jusqu'alors en gommant la technicité (50). Aussi cette direction technique est susceptible d'isoler cette proposition déviante dans le jeu complexe des interactions disciplinaires.

Toutefois, cet éveil technique en cours de définition, se transforme brutalement lors de la sévère critique médiatisée de l'éveil des années 19821983, menée par une série d'auteurs condamnant "le bonheur immédiat " des activités motivantes de l'éveil et revendiquant une instruction libératrice.

\section{DISPARITION DES ACTIVTTÉS MANUELLES}

Cette polémique conduit à la diffusion de nouveaux textes officiels fortement empreints des valeurs - conscience nationale, liberté par la connaissance, sens de l'intérêt général, volonté de participer au progrès de l'humanité (51) - dans la reconquête de l'unité nationale. Le nouveau découpage disciplinaire fait disparaître les activités manuelles et apparaître un nouveau complexe, Sciences et Technologie, dont une des missions est de contribuer au rôle assigné à l'école * de fer de lance de la modernité * afin de s'adapter aux * impératifs économiques et à la compétition internationale $*$.

L'électronique et l'informatique répondent immédiatement à ce souci d'image par la réalisation en classe de montages électroniques et l'étude des objets et systèmes informatiques (52). Ces domaines d'activités permettent en outre une approche méthodologique de la démarche technologique, démarche spécifique qui centre les études sur les objets techniques dans leur processus de genèse à la fois sur le plan technoscientifiquẹ et social. Ce contenu du cours moyen prolonge les activités du cours préparatoire et du cours élémentaire dans lesquelles demeurent la fabrication d'objets, l'usage des outils manuels, la perception du matériau tout en assurant une 
continuité avec le traitement de l'information ou les premières connaissances à propos du courant électrique, vecteur de l'information. De même, ce contenu prépare une composante de la technologie du Collège qui se centre sur le produit en proposant une lecture plus large que celle de l'objet strictement technique (53).

Parmi les activités proposées aux élèves dans les compléments au programme, dans les manuels et dans les revues pédagogiques apparaissent les deux courants complémentaires, d'une façon mineure le courant technico-technologique et d'une façon majeure le courant physico-technologique. Ce qui distingue fondamentalement ces deux orientations c'est le point de vue porté sur les « choses *; le premier s'attache à la finalité de cette réalité, à l'intégration des intentions qu'elle assure, à l'efficacité des moyens mis en œuvre, au traitement des aléas inhérents à sa concrétisation et à son fonctionnement, le second s'intéresse à la modélisation de cette réalité, à l'intégration des phénomènes qu'elle exploite ou qu'elle subit, au fondement théorique des procédés, à l'explication des aléas. La démarche de projet qui. définit cette éducation technologique met ainsi en œuvre ces nouveaux matériaux ou ces nouveaux objets, ces organes ou ces phénomènes, que sont les diodes, . résistors, transistors, moteurs ou interfaces et permet aux élèves de réaliser ou de modéliser des alarmes, des dispositifs lumineux ou des systèmes automatiques en relation avec les produits de plus en plus intelligents de l'environnement transformé par la révolution informatique.

A la date de la disparition des activités manuelles est ainsi associée la naissance de la technologie progressivement élaborée au cours de la construction de l'éveil scientifique, plus particulièrement grâce aux recherches de l'INRP (54).

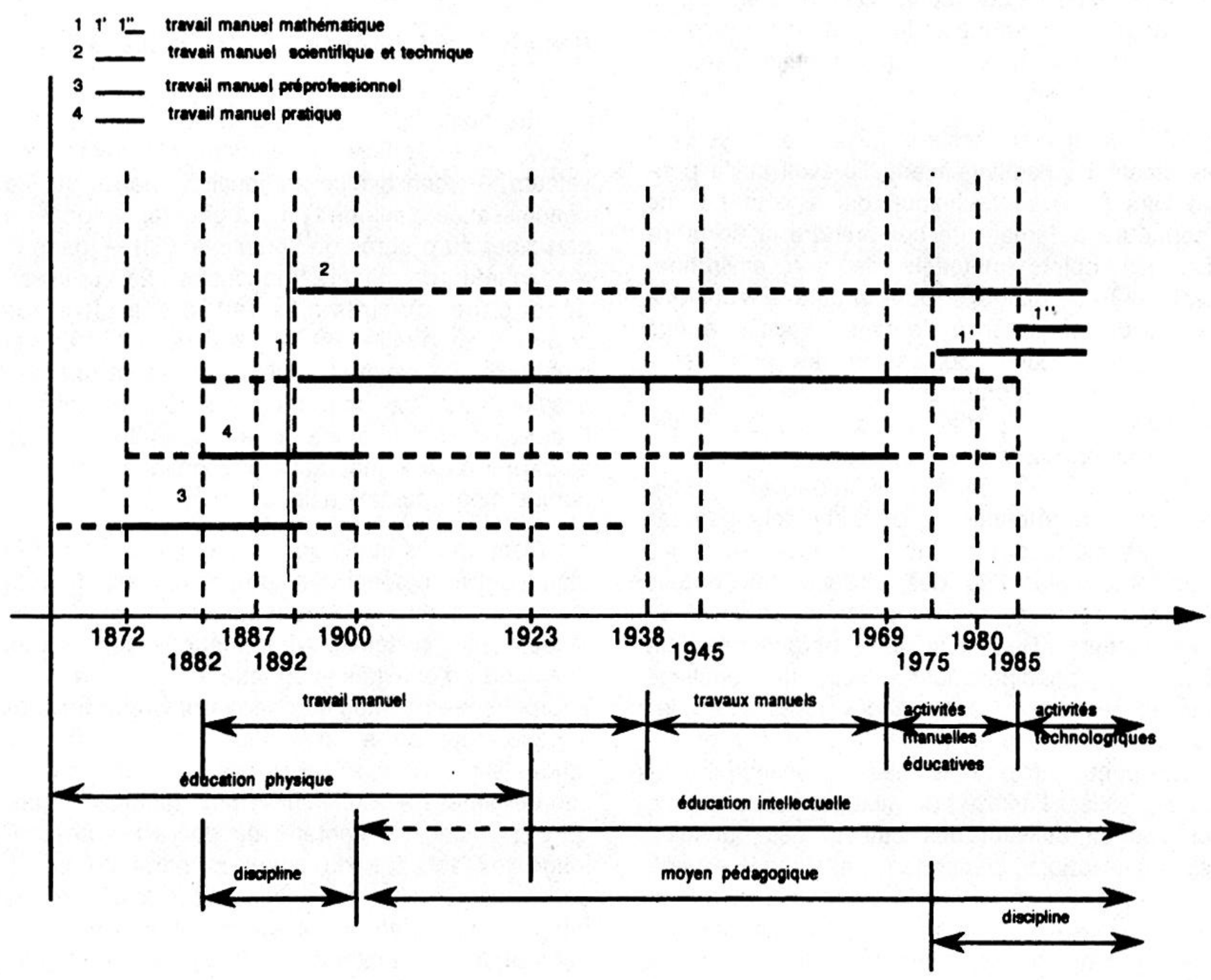




\section{PROPOSITIONS INTERPRÉTATIVES}

\section{a) Évolution générale}

L'évolution constatée peut se décrire selon les deux tendances majeures du travail manuel: la formation pratique, à dominante professionnelle ou privée et la formation scolaire à dominante scientifique, mathématique ou physico-technologique. Ce chronogramme récapitulatif montre le maintien de l'orientation mathématique (55), d'abord géométrique puis logique et algorithmique. On peut y lire aussi la mort du travail manuel préprofessionnel grâce à son attribution à un autre segment de l'instruction publique. Nous y remarquons surtout les périodes de transformations, d'abord en 1900 où la relance du travail manuel est associée au nouveau produit "le travail manuel sans atelier " et dans les années 1975, lorsque l'éveil traverse les disciplines et où se définissent les activités manuelles éducatives qui vont plus tard s'associer, se juxtaposer, s'intégrer au complexe technologie.

Cette chronologie est indissociable du mouvement général d'évolution de l'École dans ses structures et son organisation, évolution marquée d'abord par son unification et la segmentation de l'enseignement technique puis par le report de l'âge de sortie différant l'issue sociale de l'école primaire. Cette chronologie n'est pas, non plus, distincte de l'évolution de l'idée 'qu'on se fait de l'enfant. Cette modification des représentations permet de passer progressivement du petit adulte tronqué avec une main et un cil à l'enfant, entité singulière; d'une méthode catéchétique d'exercices commandés à une méthode collective mais individualisée d'activités projetées. Cette évolution s'accompagne conjointement de la complexification de l'éducation marquée par l'intégration successive des fonctions motrices $(\sim 1882)$, cognitives $(\sim 1900)$, affectives $(\sim 1950)$ et socio-affectives $(\sim 1970)$ assurant partiellement la réalisation de la trinité, corps-esprit-cœur, que souhaite accomplir l'éducation technologique.

\section{b) Conditions d'existence}

Au-delà de cette évolution globale, observer et analyser, dans une démarche explicative, le parcours d'une discipline d'enseignement depuis son institutionnalisation permet de remarquer et d'isoler les mouvances externes et les mouvances internes propres à la discipline elle-même. Le pre- mier registre d'observation s'attache à montrer l'influence des politiques éducatives et des doctrines pédagogiques alors que le second registre permet de saisir les questions spécifiques que pose une discipline.

Au plan des finalités, cette succession de formes différentes s'explique selon la focalisation plus ou moins grande sur le mot travail ou le mot manuel, dissociés lors de la rupture atelier/école. Pourtant, d'une façon permanente, l'éducation et l'instruction de l'écolier de onze ans se définissent selon ces deux directions, privilégiant tantôt le devenir de l'être social, le travailleur, tantôt le développement de la personne, l'Homme. Le choix de la première apparaît solidaire des préoccupations majeures à portée économique, une exigence des situations temporaires telles que la guerre de 1870, l'occupation ou l'inquiétude des années 1980. Par contre, les périodes d'effervescence de 1936 ou de 1968 sont à l'origine du choix de la seconde direction qui tente de promouvoir un homme nouveau. Mais cette opposition traduit plus fondamentalement la recherche du sens du travail manuel dans le conflit entre pédagogie de l'essence et pédagogie de l'existence (56), entre idéal et vie. L'humanisme travailliste, cette éducation intégrale, en est la première expression à la fin du XIX siècle dans l'école primaire populaire. L'éducation nouvelle puis l'éveil reposent sur la même tentative d'harmonisation de l'opposition, actualisée, entre développement personnel et adaptation sociale plus particulièrement analysée par les sociologues (57). Le travail manuel, ancré radicalement sur les références sociales, révèle, sans doute plus que toute autre pratique scolaire, cette recherche de définition du produit de l'éducation. La technologie participe, elle aussi, à ce débat suggérant une éducation technologique proposant développement de la personne et formation sociale. Mais l'originalité de cette dernière réside, au delà d'une visée professionnelle, dans la contribution à la formation du citoyen (58), jusqu'alors absente ou assimilée à l'éducation morale exercée par le soin, le respect des règles qui permet d'imposer la droiture au penchant sinistre.

Parallèlement à cette interrogation liée aux finalités, la lecture des prescriptions fait apparaître deux orientations dans les objectifs et les contenus qu'ils sous-tendent. Savoir pratique et savoir théorique, savoir-faire et savoir sont associés dans tous les textes. L'objet fabriqué ou analysé 
est alors prétexte à mise en cuvre d'outils, d'engins, de procédés et à élaboration conceptuelle.

Ces deux axes d'analyse permettent de localiser les différents styles de travail manuel sur un diagramme socio-didactique. Primat de l'enfant et de son développement personnel, primat de l'enjeu sociétal, focalisation sur un savoir pratique ou un savoir plus théorique permettent de délimiter quatre secteurs parcourus par le travail manuel.

Cette représentation indique la localisation privilégiée du travail manuel dans le quartier caractérisé par la relation de l'enfant avec un savoir théorique, ce qui le légitime à l'école (59). Dans le questionnement de cette légitimité, il serait vain de sous-estimer l'influence des valeurs de l'École, il serait illusoire de masquer la logique des acteurs qui ne diffusent que les pratiques culturelles auxquelles ils adhèrent et il serait naîf d'ignorer que la demande des familles n'est pas une formation scolaire au travail car la libération de l'individu par et grâce à l'École ne passe pas par des apprentissages, si minimaux soient-ils, de la sphère sociale. Mais ce point de vue axiologi- que ne permet pas d'expliquer le maintien du travail manuel, les absorptions, les rejets et les adaptations des éléments de son contenu. Son existence à l'École, le sens qu'il prend au cours moyen, la légitimité sociale qu'il a, se construisent, aux différentes périodes, par l'intermédiaire des disciplines installées, selon deux modalités. La première consiste à le rendre légitime grâce au contenu des autres disciplines ce qui lui donne son aspect accessoire en étant l'appendice de celles-ci. La seconde est une légitimation scolaire par sa fonction de régulation dans l'organisation pédagogique ce qui lui donne son aspect ludique permettant de ponctuer l'effort de l'écolier, de a régénérer l'intelligence * selon l'expression de Schmitt. Ainsi, son existence en classe, pour laquelle ses supports matériels exigent un effort d'organisation, ne semble envisageable que si le sacrifice qu'il impose équilibre les avantages qu'il apporte dans l'un ou l'autre de ces deux aspects.

Mais ces conditions d'existence signalent aussi les limites de son identité scolaire. La déviance manifeste du travail manuel à l'école, conçu très tôt comme un travail manuel d'école pour les

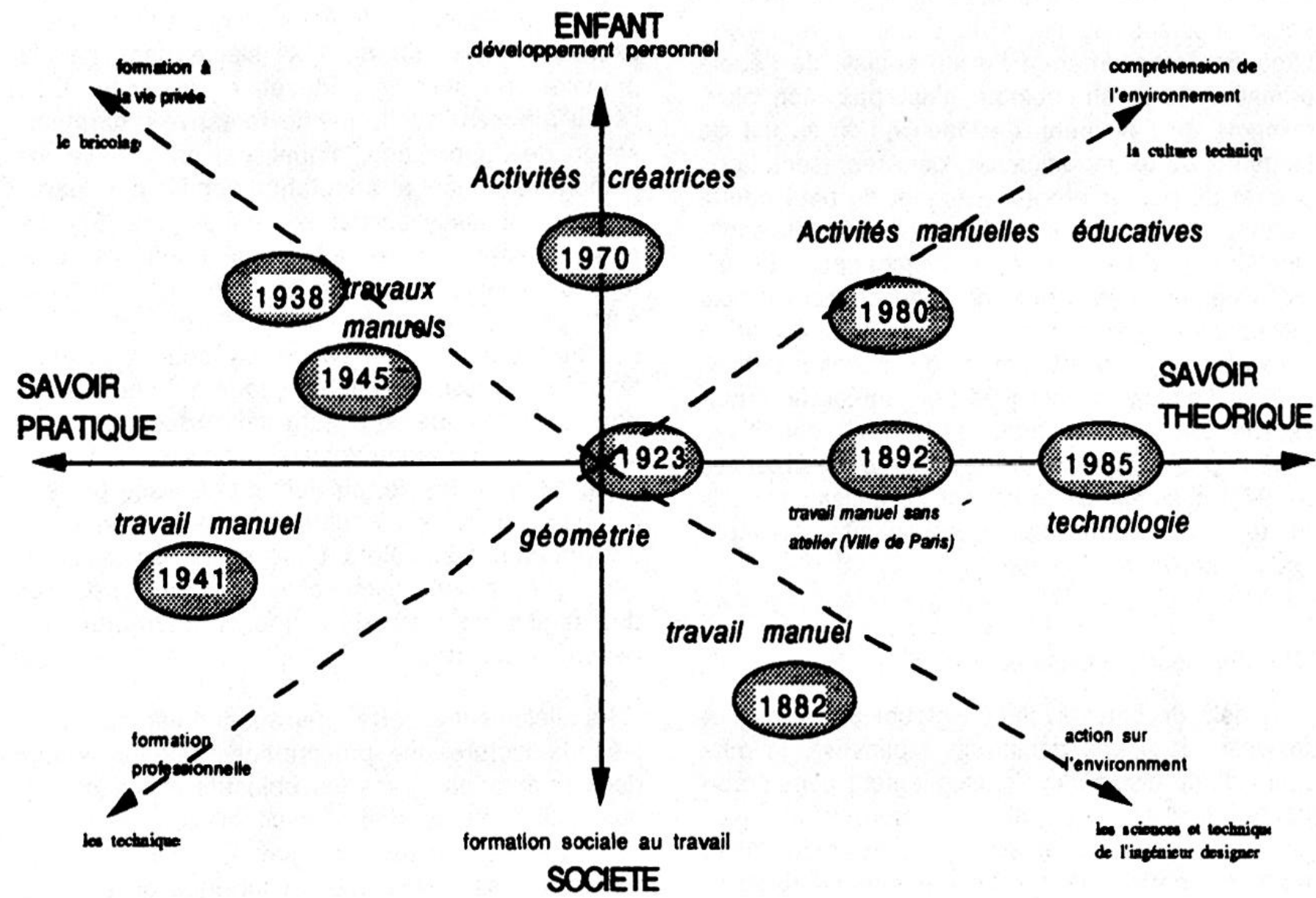


enfants (articulé avec celui de la maison d'enfants), correspond à la référence professionnelle comme en témoigne la proposition inacceptable qu'a pu être, à l'époque, la méthode de la ferblanterie. Au cours de ce siècle, la dimension technique, la référence à l'atelier ou à l'entreprise, au métier ou à la profession, à la production est, par une série d'évitements, écartée de l'école primaire. La séparation précoce de l'atelier et de l'école, facilitant l'absence généralisée de lieu spécialisé, a ainsi favorisé l'association du travail manuel à l'éducation intellectuelle en privilégiant la posture assise de l'écolier, déterminante dans le choix d'exercices ou d'activités. Dès lors, il doit prendre appui sur les références indiscutables de l'école, avec lesquelles le contenu entretient un lien de parenté. Pour ce qui concerne le travail manuel au cours moyen, la référence interne privilégiée, le point d'ancrage majeur des contenus, sont les sciences. Le travail manuel géométrique illustre notamment cette articulation qui permet une évocation allusive au métier avec un cahier, du papier et un crayon et une progression linéaire sur les cycles.

Cet ancrage révèle aussi l'insuffisance d'une référence interne autonome. En effet, la référence manuelle, malgré la permanence du souhait de développement de l'habileté, demeure l'attribut des classes enfantines, préparatoires et élémentaires en relation avec la gestualité de l'écriture. Au delà, cette référence apparaît très vite caduque dans une visée professionnelle de plus en plus lointaine et de moins en moins opératoire, et a-scolaire dans la dimension privée de la formation sociale. De plus cet aspect s'avère, très tôt exclu de l'école, car particulièrement adapté et bientôt dévolu et réservé à l'éducation des « arriérés $\approx(60)$. La référence technique, quant à elle, est ignorée car trop récente dans les préoccupations de formation générale et jugée déraisonnable car supposée sans raison.

Néanmoins les tentatives de définition d'une spécificité, la recherche d'une référence scolaire autonome ont été au cceur des préoccupations. Les différents discours, de Pestalozzi à la COPRET, sont construits selon le fil conducteur des apprentissages à promouvoir afin de ne pas enseigner une spécialisation insensée car prématurée, ni dispenser un savoir fermé car immédiatement obsolète. ABC technique, connaissance commune à tous les métiers, habileté et goût, démarche technologique, culture technique per- mettent, au fil de l'évolution de l'environnement matériel, de penser des activités significatives. Toutefois, cette spécificité recherchée se trouve continuellement absorbée par les disciplines. La technicité, quintessence des techniques, cette ruse de l'homme pour produire, pour transformer, pour vendre est métamorphosée en un discours sur la technique, une formalisation rationnelle qui se substitue à la pensée en acte, qui l'anticipe - pour l'éviter. Jamais le travail manuel ne se définit dans une perspective de développement de la technicité, jamais il ne s'associe à production technologique enfantine car l'école ne tolère ces manifestations que dans le domaine esthétique.

Protection de l'enfant, refus d'un déterminisme social fatal et/ou conformation aux rites de l'école ont conduit les prescripteurs à la proposition d'enseignements qui ne conservent que la connaissance et la compréhension de l'environnement en détournant le domaine de l'action. Le travail manuel se confond alors avec pédagogie de l'action ou du concret. Si à l'école, technique se confond souvent avec action, l'efficacité de l'action qui donne le sens des techniques industrielles ou commerciales n'est jamais centrale.

\section{COHÉRENCE ET CONFLIT DE RÉfÉRENCES}

Toutefois dans ce quartier de la formation générale, nous avons noté des variations des formes scolaires du travail manuel, différents styles qui, malgré leur apparence ne sont pas des feux de paille témoins des modes et de l'esprit du temps. En effet cet itinéraire illustre le processus de maintien de ce travail manuel, éducatif car enseigné, transposé car scolarisé. Le contenu oscille alors entre les orientations extrêmes et ses dérives éventuelles que la succession des méthodes semble corriger. Ainsi, le professionnalisme de la méthode des éléments techniques (1872) est adaptée par la méthode des objets utiles (1887) dont l'utilitarisme est corrigé par la méthodes des éléments géométriques (1891) au rationnel compensé par la méthode des objets attrayants (1923) dont l'approximation est structurée par la méthode des éléments logiques (1975) au formalisme que tempère la méthode du projet technique (1985) (61).

Mais plus qu'une succession de méthodes, il s'agit d'une alternance de méthodes traduisant la difficulté d'intégration dans le système scolaire 
des pratiques de l'environnement, la difficulté d'articuler les pratiques de référence, dans leurs nuances et leur évolution, avec les pratiques scolaires. Ces méthodes ne sont en fait qu'une alternance des références scolaires et des références externes. La méthode des objets utiles, des objets attrayants, du projet technique sont des transpositions de pratiques privées ou professionnelles, elles s'opposent aux méthodes des éléments techniques, géométriques ou logiques fondées sur la construction linéaire des apprentissages en exercices gradués. Cette alternance du discours pédagogique apparaît comme une recherche de cohérence de cet ensemble de pratiques, dans le conflit de références qu'il pose. Dans cette recherche d'équilibre entre un travail manuel d'école et un travail manuel authentique, les références externes semblent ressourcer les pratiques lorsqu'elles ont perdu leur sens initial, lorsque les contenus deviennent, par excès de traitement scolaire, intégrés dans les activités qui relèvent alors d'autres champs disciplinaires. Le travail manuel sans atelier a ainsi peu à peu éloigné définitivement et irréversiblement la référence externe pour ne pouvoir avoir du sens que dans les références internes de l'école. Le travail manuel attrayant a permis alors de lui redonner du sens en référence à des pratiques privées. Mais là encore, son excès a impliqué son écart, sa déconnexion de l'école. Inversement sa scolarisation excessive dans la méthode des éléments géométriques ou logiques conduit à la même perte de sens. Telle semble être la dynamique du processus de scolarisation du travail manuel et d'intégration didactique de cet ensemble de pratiques sociales, évitant sa juxtaposition fonctionnelle et garantissant, malgré le pluriel de ses appellations scolaires, la singularité de sa proposition.

\section{Joël Lebeaume LIREST-GDSTC ENS Cachan Université d'Orléans \& IUFM Orléans-Tours}

(1) Étude effectuée dans le cadre du DEA de didactique des disciplines sous la responsabilité de J.L. Martinand : Cent ans do travail manued pour los garcons de cours moven, UER didactique des disciplines, Paris VII, 1989. Elle a été poursuivie et constitue la première partie d'une these : Cont ans de travall manuel pour l'scole elementairo-Aspects ofdactiques, Université Paris-Sud, 1993.

(2) Histoire do PÉducation, $n^{\circ}$ 38, Paris, INRP, 1988

(3) Remarque : en novembre 1882 la création de l'école normale supérieure de travail manuel a permis la formation spécifique d'enseignants mais aux termes du décret du 4 septembre 1884, elle a été réunie à celle de St-Cloud à dater du $1^{\text {er }}$ octobre.

(4) Hébrard J., La scolarisation des savoirs élémentaires à l'époque moderne, Mistoire de P'Éducation, Paris: INRP, 1988, $n^{\circ} 38$, p. 10.

(5) Arnaud P., Contribution une histoire des disciplines d'enseignement : la mise en forme scolaire de l'éducation physique, Roves Francalos de Pcodagogio, Paris: INRP, 1989, p. 29-34.

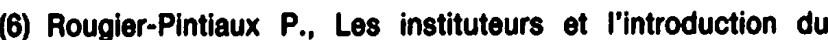
tavail manuel dans les écoles primaires de garcons du $X X^{\circ}$ siecle, Revis Francalies de Sociologie, XXIX, Paris, 1988, p. 275-292. Selon l'auteur, cette interprétation scolaire des pratiques sociales du travail, préconisée par la hiérarchie administrative, a été renforcée par la revendication des instituteurs (réunis au congress du Havre, 1885), de leur professionnalisme de la transmission de tout savoir, ecartant ainsi l'intervention des maitres-ouvriers.

(7) Remarque : L'expression * éducation harmonique * se définit selon la solidarité des trois ondres des facultés humaines : la main, l'esprit, le ccour.

(8) Legoux Y., Du compegnon au technicien, Paris : Technique ot Vulgarisation, 1972.
(9) Cette formule est utilisée par Salicis dans * l'enseignement manuel et professionnel en Allemagne w. L'origine de Salicis, polytechnicien, capitaine de frégate, explique cette référence militaire qu'il nuance * la jeune armée du travail . (lettre au ministre du 29 mars 1886, p. 22, A.N. F17 9674). Le * succès économique sur les marchés internationaux * (instruction spéciale 1886) lui semble solidaire d'une formation visant à terme un * ouvrier non seulement habile mais instruit * (instruction pour l'enseignement manuel dans les écoles primaires, Paris, Chaix, 1885, p. 7) que recouvre la formule * bras intelligents * utilisé par Planty (A), Coure de trevail manuel, Paris, Gedalge, 1887, préface p. II.

(10) Corbon revendique l'origine de l'introduction des exercices manuels à l'école primaire dans sa lettre au ministre du $1^{\text {"w }}$ novembre 1885.

(11) Corbon A., Rapport a M. lo Sensteur, Prtet de la Solne sur les exerelces manudis dans les cooles primairea condidértes commo complément de l'seducation, Paris: Charles de Mourgues, 1880, p. 12.

(12) Panthier A., Enquito hiatoriqus aur Penseignement manuel dane les ćcoles non techniques, Paris : Imprimerie nationale. 1906, p. 54.

(13) Remarque: le système du Kindergarten propose deux séries d'activité ludiques utilisant des matériaux : les dons et les occupations. Les dons consistent à manipuler, à construire des corps, des surfaces, des lignes ot des points. Les occupations apparaissent ensuite faisant place à l'enseignement systématique des connaissances * dissimulées * sous les dons. Points, lignes, surfaces et volumes sont abordés par des activités de piquage, de couture, tissage, pliage, cartonnage, modelage...

(14) Montessori M., Psdagogie scientificuve, tome 1, La maison des enfants, Paris : ESF, 1970 (Édition du centenaire). 
(15) Pestalozzi. Comment Certrude instruit ses enfants, Albeuve, Castella, 1985, p. 203, traduction par Soëtard (M.) de l'édition originale Wie Gertrud ihre kinder lerht, Bern et Zürich, Heinrich Gessner, 1801.

(16) Schmitt E., La podagogio du travail manuel, Paris : Alcide Picard et Kaan, 1888.

(17) Concept didactique introduit par Martinand (JL.), Connalture of transformer la matiore, Berne: Peter Lang, 1986.

(18) Ognier P., L'école républicaine françalse of $80 s$ miroirs, Berne : Peter Lang, 1988, p. 91.

(19) Leblanc R., Les travaux manuels dans les Écoles Normales d'Instituteurs, Paris : Revve Pédegogique, 1891, I, p. 9 et p. 233-244.

(20) Publié de juillet 1888 à septembre 1890, Paris, Librairie Gedalge.

(21) Prost A., Histoire de l'ensoignement en France (1800-1867), Paris : Armand Colin, 1968.

(22) Cette revue est publiée aux éditions Nathan, le premier numéro est daté du $1^{\text {er }}$ octobre 1922. Elle devient le * Magazine scientifique illustró "à partir du $1^{\text {er }}$ mars 1928 puis prendra le nom de * Scola * jusqu'en 1934. L'examen de cette revue montre, comme l'évolution du titre le suggère, un point de vue scientifique privilégié.

(23) Remarque : les auteurs réguliers des articles des six premières années, P.E. Marcant, A. Plicque, E. Réau, $M^{m e}$ Chanticlaire, sont aussi les auteurs de livres, de matériel, d'outillage, de cahiers édités par Nathan et auxquels la revue renvoie.

(24) Plicque A., Lo travall manuel attreyant a l'ścole primaire, Éducation de l'cill et de la main, Paris : Nathan, 1922.

(25) Kula C., Bocquillone E., Pour lo bonheur de nos enfants, la ńforme de P'oducation natioanle, Paris, Dunod, 1933.

(26) Référence à la devise de Jeanne d'Arc qui semble étre la formule de l'époque pour glorifier le travail comme l'indique le discours prononcé par M. Labbé, directeur de l'Enseignement technique lors de la fôte du travail à Paris, le $18 \mathrm{dé}-$ cembre 1926: “... Voilà ce que nous fêtons et je regrette qu'il ne puisse monter de cette jeunesse qui se presse devant moi, un chour solennel qui chanterait au travail un hymne où l'on entendrait les vieux mots français, la plus belle des devises: * vive labeur *!

(27) Création au début de l'année 1906 au 52, rue des Épinettes à Paris.

(28) Bocquillone E., Do PÉcole a Pateller, Paris, Baudinière, 1944, p. 42.

(29) Cunéo M., Godier A. Vado-mecum pour les acthittes diriges et l'stude du millou, Paris: Nathan, 1948, $3^{\circ}$ ed., p. 6.

(30) Instructions de 1938, p. 4.

(31) Bien que dans les textes des années 1940 dessin et travail manuel soient juxtaposés (dessin travail manuel en 1938, dessin ou travail manuel en 1947), il s'agit essentiellement d'un regroupement horaire plus que d'une assimilation de contenus ou d'activités car les instructions demeurent distinctes.

(33) Cousinet R. La pédagogie du travail manuel, Paris, L'́cole Nouvelve Frangalse, $n^{\circ} 30,1950$, p. 35-39.

(34) Calmy G., Lo travail manuel do 4 d 7 ans, Paris, 1961.

(35) Plaisance E., L'Coole matemelle en France depule ta fin do la seconde guerro mondialo, thèse, UER Sciences de l'Éducation, Paris V, 1984.

(36) Circulaire ministérielle du 14 avril 1953 in Encyclopédie pratique de l'éducation en France, IPN MEN, Paris, 1960, p. 570.

(37) Selon Gloton R., 1969.

(38) Remarque: cette réflexion aboutit a la publication d'un ouvrage en deux volumes: Collectif des PEN, Comment pratiquer les activites manuelles 6ducatives a l'école maternelle et à l'école EKémentaire, Marseille, CRDP, 1978.

(39) Cette relation est argumentée dans une perspective génétique au plan de la psychologie et de l'anthropologie, la main qui pense, le primat de l'action sur la pensée sont les thèmes de la dialectique du geste et de la parole.

(40) Schneider J., Los actuvites manuelles ot l'́rvell de la pensé, op. cit., note 38, p. 9.

(41) Voir notamment Bresson M., De Schonen S., Pratiques de l'espace, film $16 \mathrm{~mm}$, Service du film de la recherche scientifique, Paris, 1980.

(42) Selon définition in Goldschager L. et Lister $A$. Informatique ot Agorithmique, traduit par Sumpf V., Inter-Éditions, 1981, p. 16.

(43) Dumont M. et Pasquis F., Mathomatique pour la tote of les mains, Paris, Cedic-Nathan, 1979.

(44) Cette caractéristique est développée par Géminard L., Loglque et Technologio, Dunod, Paris, 1970.

(45) voir disciplines majeures, disciplines mineures in Léon A., Introduction a l'histoire des felts socucetife, Paris: PUF, 1971.

(46) Remarque : ce courant transforme également l'éducation gestuelle en psychomotricité à l'école maternelle.

(47) Collectif des PEN, Activites manuelles educatives au cours moyen, tome 3, Marseille: CRDP, 1986, p. 108.

(48) Fiches d'accompagnement 1981-82 et fiches du Journal des Instituteurs, Nathan.

(49) Op. cit. p. 8.

(50) Martinand J.-L., Culture et technicité dans l'enseignement obligatoire, Actes du colloque "Culture technique et formation $\nsim$, Nancy: PUN, 1991, p. 54-56.

(51) Isambert-Jamati V., Les primaires, ces incapables prétentieux, Revue françalise de pedagogie, $n^{\circ} 73,10-11-12 / 85$, Paris, INRP.

(52) Remarque : cette dernière est la plus retentissante soutenue par un plan d'équipement et de dotation matérielle (valises Technologie), adjointe à une formation massive des maîtres et supervisée par une infrastructure décentralisée (plan IPT, animateurs de réseaux...).

(53) Cf. rapport COPRET, 1985.

(54) Voir notamment: Activités d'éveil scientifiques à l'école élémentaire. Initiation physique et technologique. Recherches Pédagogiques, $\pi^{\circ} 74$. Paris : INRP. 1975.

(55) Remarque : le graphique distingue ces variations, géométrique (1), algorithmique (1') traduites sous forme de procédures dans l'écriture des programmes de * robotique * (1").

(56) Suchodolski B., La pedagogie et los grands courants philosophiques, Paris : Éditions du Scarabée, 1960.

(57) Voir notamment Isambert-Jamati, Culture technique of critique socialo l'ccole Komentaire, Paris, PUF, 1984.

(58) Cf. Copret remarque importante p. 5.

(59) Remarque : la problematique du travail manuel féminin, au moins pour la première partie du $X X^{\circ}$ siècle est différente, dans sa visée d'éducation ménagère qui prend essentiellement référence sur la vie privée.

(60) Pascale Rougier-Pintiaux remarque le thème choisi pour le premier examen du certificat d'aptitude a l'enseignement des enfants arriérés qui est * l'importance du travail manuel dans l'éducation des arriérés * (cf. Revie Pćdagoglones, 1910, 2 semestre), op. cit. p. 2.

(61) Remarque : les dérives de cette méthode sont ou bien le dressage industriel par l'application répétée et systématique d'une démrache ou bien l'allusion produite par un discours de plus en plus distant de la réalité. 